\title{
Psychological distress and risk of accidental death in the general population
}

\author{
G. David Batty, Emmanuel Stamatakis and Steven Bell
}

\section{To the Editor -}

While accidents are a major cause of disability and mortality worldwide, their aetiology is not well understood. Risk factors appear to include socioeconomic deprivation, lower cognition, and being male, older, and unmarried/living alone. ${ }^{1-3}$ Investigators have also shown that severe mental illness is associated with an elevated risk of accidents, ${ }^{2}$ however, these observations are exclusive to individuals with mental disorders serious enough to warrant hospitalisation ${ }^{4}$ or referral to an outpatient clinic. To the best of our knowledge, there has been no examination of the link between lower levels of distress and subsequent risk of accidents. Strong prima facie reasons to anticipate such a relationship include the symptoms of fatigue, poor concentration, and sleep disturbance, that characterise even moderately distressed individuals, which may impact unfavourably on decisionmaking, risk perception, coordination, and response time, so precipitating accidents.

Described in detail elsewhere, ${ }^{5}$ we used data from sixteen independent, geographicallyrepresentative surveys with mortality surveillance (the Health Survey for England $[N=13]$ and the Scottish Health Surveys [N=3]). Combining these studies in the context of an individual-participant meta-analysis resulted in a total of 193,873 participants, $166,606(86 \%)$ of whom had data on age, sex and psychological distress. Psychological distress was measured using the 12-item General Health Questionnaire (GHQ-12) which contains items principally concerned with symptoms of depression and anxiety. The sensitivity (0.70) and specificity (0.80) against standardized psychiatric interview is acceptably high. ${ }^{6}$ Study members also reported if they had even been diagnosed with a psychiatric disorder and if they had used prescribed psychotropic medications. Hazard ratios were computed using Cox proportional hazard models in which we accounted for between study variation using a shared frailty parameter. We used fractional polynomials to estimate the best-fitting dose-response curve. 
In analyses of baseline data, we found that a higher prevalence of both self-reported psychiatric morbidity and psychotropic medications was apparent in the more psychologically distressed study members (supplemental figure 1). For instance, in people in the most distressed group (GHQ-12 score 12$), 31 \%$ reported using psychotropic medications, whereas in the least distress group (GHQ12 score 0$)$ it was $2 \%$.

A mean duration of 9.5 years of follow-up of 166,606 people (91,528 women) gave rise to 340 deaths ascribed to all accidents combined. In figure 1 we show that, following multivariable adjustment, an increase in the rate of accidental deaths occurred at a distress scores of above 3. After excluding study members with either self-declared psychiatric morbidity or use of psychotropic medications (111,848 people; 218 accidental deaths), hazard ratios became elevated at the lowest level of distress symptoms. A test for trend indicated a dose-response effect across the full range of distress scores ( $\mathrm{p}$-value for trend $\leq 0.001$ ), with a one-standard-deviation-increase in psychological distress associated with an increase in risk of accidents after multiple adjustment $(1.17 ; 1.04,1.33)$. In supplemental table 1 we present the risk of accidents according to standard categorisation of psychological distress ('asymptomatic', 'sub-clinical symptomatic', 'symptomatic', 'highly symptomatic').

In this, the first population-based study in the context of psychological distress symptoms and accidental death, we found that accident rates were raised even in people with low levels of distress. That we were also able to show that known risk factors for accidents - gender (male vs. female: $1.80 ; 1.45,2.23)$, older (per year increase in age: $1.05 ; 1.05,1.06)$, unmarried/living alone (1.97; $1.58,2.45$ ), and of lower social status (manual vs. non-manual social class: $1.48 ; 1.18,1.85$ ) - were replicated herein (HRs are age-and sex-adjusted) gives us some confidence in our novel results for psychological distress (supplemental table 2). With this association being stepwise and seemingly 
robust to the adjustment of a series of confounding factors, implicates other, as yet unknown, mechanisms in this relationship. 


\section{References}

(1) Batty GD, Gale CR, Tynelius P, Deary IJ, Rasmussen F. IQ in early adulthood, socioeconomic position, and unintentional injury mortality by middle age: a cohort study of more than 1 million Swedish men. Am J Epidemiol 2009; 169(5):606-615.

(2) Crump C, Sundquist K, Winkleby MA, Sundquist J. Mental disorders and risk of accidental death. Br J Psychiatry 2013; 203(3):297-302.

(3) McCartney G, Russ TC, Walsh D, Lewsey J, Smith M, Smith GD et al. Explaining the excess mortality in Scotland compared with England: pooling of 18 cohort studies. J Epidemiol Community Health 2015; 69(1):20-27.

(4) Hiroeh U, Appleby L, Mortensen PB, Dunn G. Death by homicide, suicide, and other unnatural causes in people with mental illness: a population-based study. Lancet 2001; 358(9299):2110-2112.

(5) Russ TC, Stamatakis E, Hamer M, Starr JM, Kivimaki M, Batty GD. Association between psychological distress and mortality: individual participant pooled analysis of 10 prospective cohort studies. BMJ 2012; 345:e4933.

(6) Aalto AM, Elovainio M, Kivimaki M, Uutela A, Pirkola S. The Beck Depression Inventory and General Health Questionnaire as measures of depression in the general population: a validation study using the Composite International Diagnostic Interview as the gold standard. Psychiatry Res 2012; 197(1-2):163-171. 


\section{Conflicts of Interest and Financial Disclosures}

None to declare.

\section{Acknowledgements}

SB is supported by grants from the European Research Council and the UK Medical Research Council/Alcohol Research UK. 
Figure 1. Dose-response association between psychological distress score and risk of accidental death: Individual-participant data from a meta-analysis of 16 general population-based cohort studies
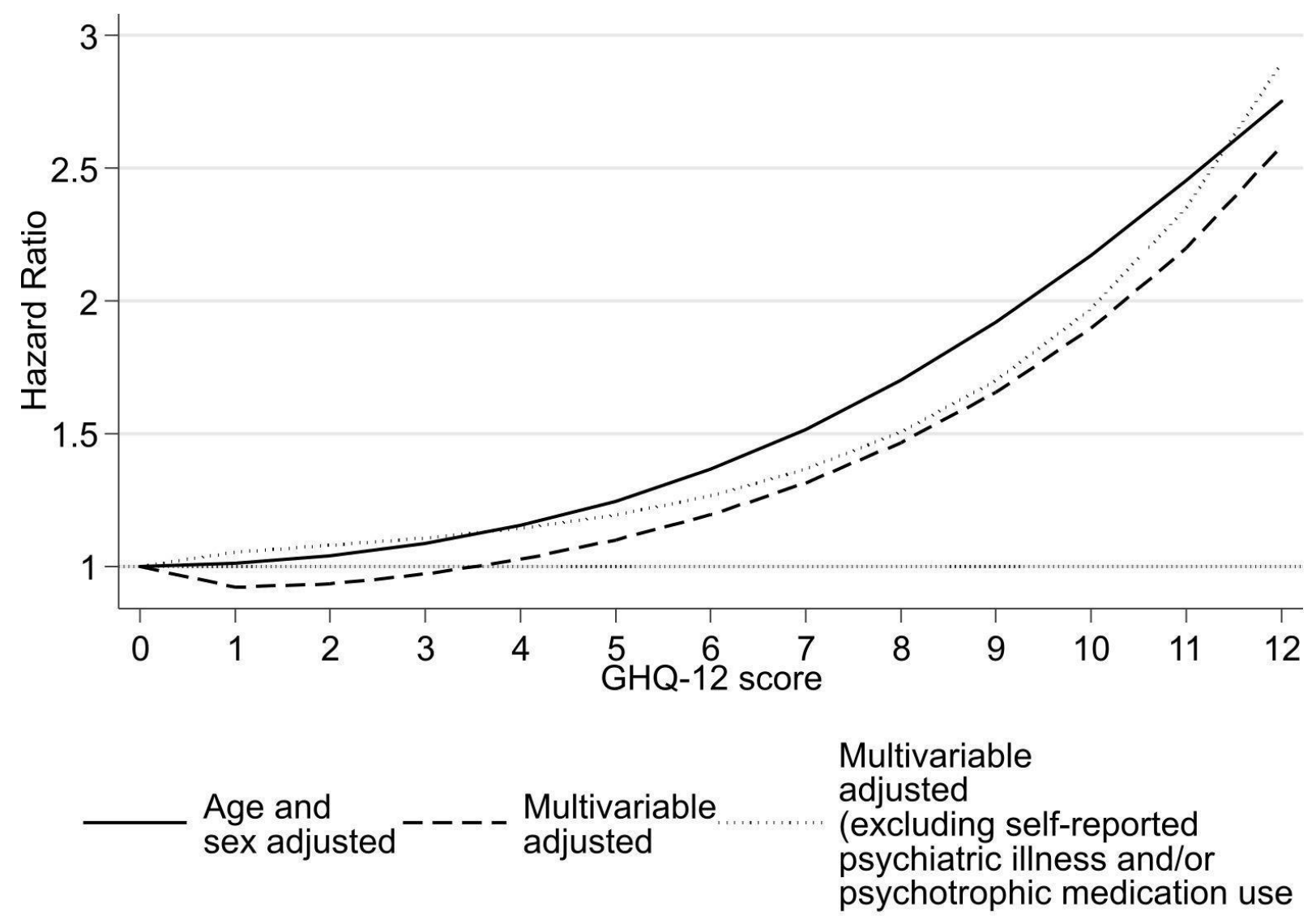

Underlying unintentional external deaths were those deaths where the underlying cause of death was external (ICD-9 codes $800-999$ and ICD-10 codes V00-Y98) and had no mention of the ICD codes indicating an intentional motivation (ICD-9 codes E950-E959 and E980-E989 or ICD-10 codes X60-X84 and Y10-Y34).

Multiple adjustment is adjustment for: age, sex, socioeconomic position (manual, non-manual occupation), frequency of alcohol consumption (never, former, occasional,

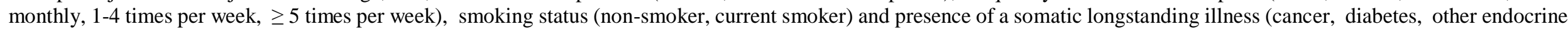
disorders, cardiovascular disease, hypertension, respiratory disease, and any other non-mental health condition). 
Supplemental Table 1. Hazard ratios (95\% confidence intervals) for the relation of psychological distress severity with accidental death: Individual participant data meta-analysis of 16 general population-based cohort studies

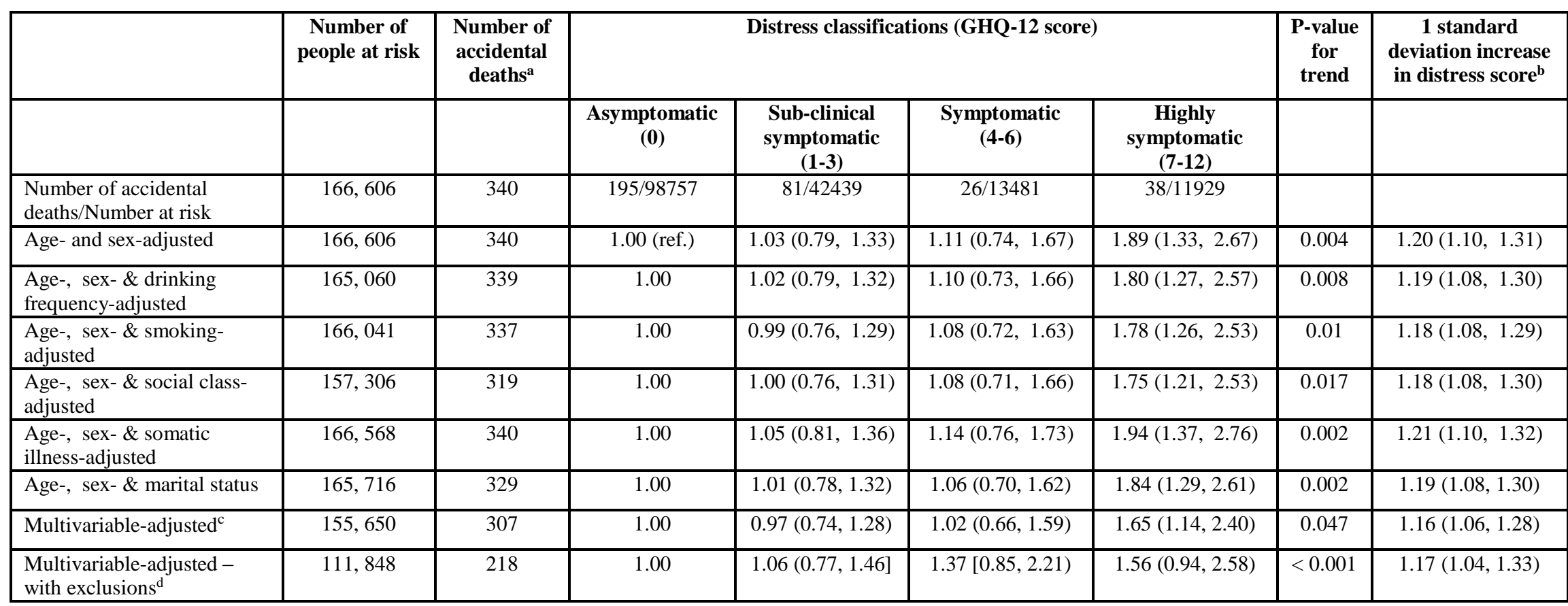

${ }^{a}$ Underlying unintentional external deaths (ICD-9 codes 800-999 and ICD-10 codes V00-Y98 - all deaths where the underlying cause of death was external and had no mention of the ICD codes indicating an intentional motivation [ICD-9 codes E950-E959 and E980-E989 or ICD-10 codes X60-X84 and Y10-Y34]).

${ }^{\mathrm{b}}$ Based on sex-specific standard deviations.

${ }^{\mathrm{c}}$ Multivariable-adjusted effect estimates are adjusted for: age, sex, socioeconomic position (manual, non-manual occupation), marital status, frequency of alcohol consumption (never, former, occasional, monthly, 1-4 times per week, $\geq 5$ times per week), smoking status (non-smoker, current smoker) and presence of a somatic longstanding illness (cancer, diabetes, other endocrine disorders, cardiovascular disease, hypertension, respiratory disease, and any other non-mental health condition).

${ }^{\mathrm{d}}$ Excluding people with diagnosed mental illness and use of psychotropic medication 
Supplemental Table 2. Relation of study covariates with accidental death: Individual participant data meta-analysis of 16 general population-based cohort studies

\begin{tabular}{|c|c|c|c|}
\hline & $\begin{array}{c}\text { Number of } \\
\text { people at risk }\end{array}$ & $\begin{array}{c}\text { Number } \\
\text { of } \\
\text { accidental } \\
\text { deaths }\end{array}$ & Hazard Ratio $\left(95 \%\right.$ CI) $^{b}$ \\
\hline Age (per year increase) & 166,606 & 340 & $1.05(1.05,1.06)$ \\
\hline Sex (female is referent) & 166,607 & 341 & $1.80(1.45,2.23)$ \\
\hline Drinking frequency & 165,060 & 339 & \\
\hline Never drank & & & $1.28(0.82,2.00)$ \\
\hline Ex-drinker & & & $1.55(0.99,2.43)$ \\
\hline Occasional (less than monthly) & & & $1.03(0.73,1.45)$ \\
\hline Monthly ( 1 to 2 times per month) & & & $1.04(0.72,1.52)$ \\
\hline 1-4 times per week & & & 1.00 (ref) \\
\hline$\geq 5$ times per week & & & $1.27(0.96,1.68)$ \\
\hline Smoking Status & 166,041 & 337 & \\
\hline Non-smoker & & & 1.00 (ref) \\
\hline Current smoker & & & $1.84(1.45,2.34)$ \\
\hline Manual social class & 157,306 & 319 & \\
\hline No & & & 1.00 (ref) \\
\hline Yes & & & $1.48(1.18,1.85)$ \\
\hline Somatic illness & 166,568 & 340 & \\
\hline No & & & 1.00 (ref) \\
\hline Yes & & & $0.90(0.72,1.12)$ \\
\hline Marital status & 165,716 & 329 & \\
\hline Married/cohabiting & & & 1.00 (ref) \\
\hline Single, divorced or widowed & & & $1.97(1.58,2.45)$ \\
\hline
\end{tabular}

All hazard ratios are adjusted for age and sex, except age which is sex-adjusted only, and sex which is age-adjusted only 
Supplemental Figure 1. Baseline psychiatric morbidity and psychotropic medications according to psychological distress $(\mathrm{N}=\mathbf{1 6 6 , 5 9 3})$
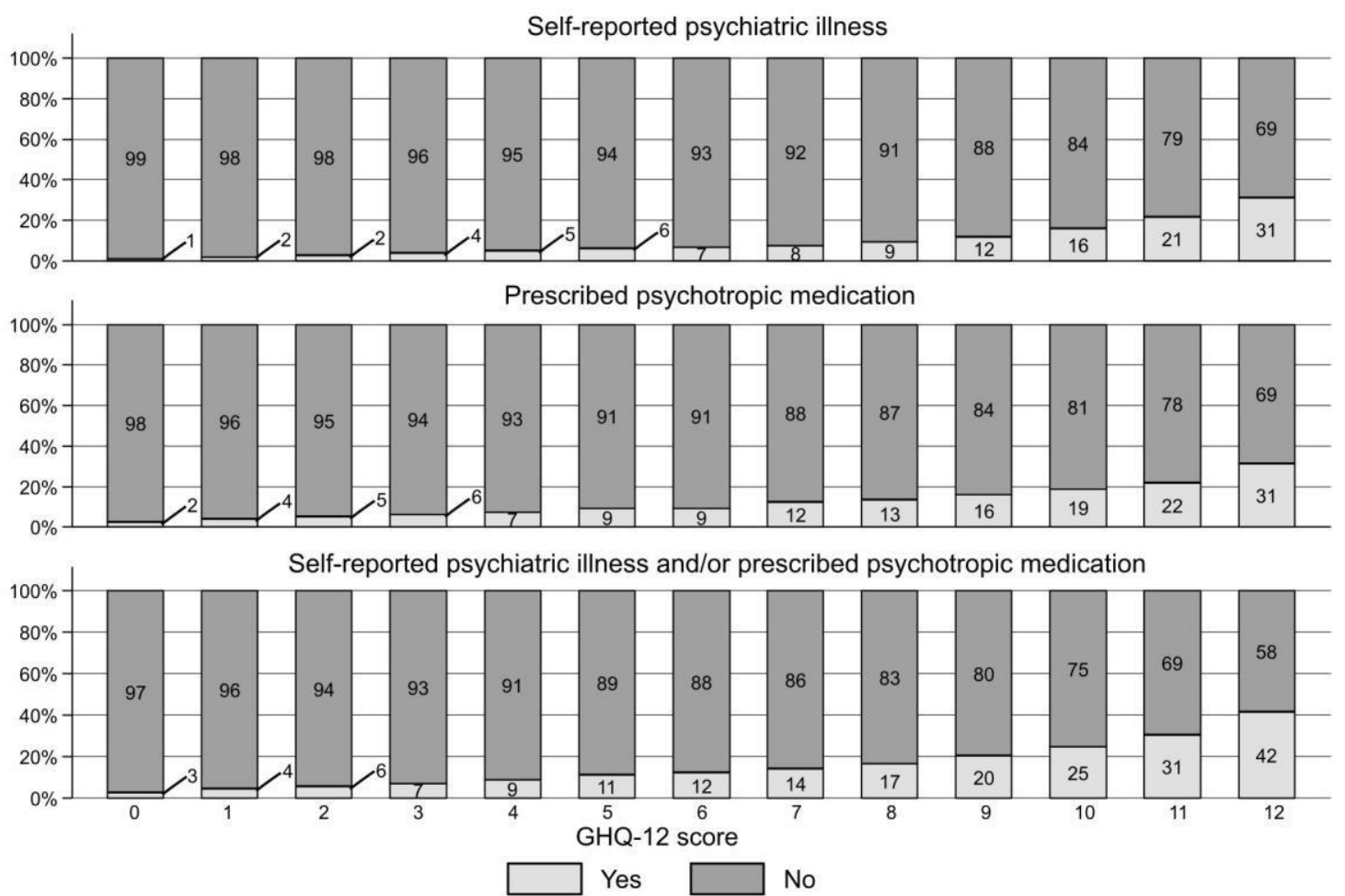